\title{
Perturbative and non-perturbative studies with the delta function potential
}

\author{
Nabakumar Bera, ${ }^{1}$ Kamal Bhattacharyya, ${ }^{2}$ and Jayanta K. Bhattacharjee ${ }^{1, *}$ \\ ${ }^{1}$ Department of Theoretical Physics, \\ Indian Association for the Cultivation of Science, Jadavpur, Kolkata 700 032, India \\ ${ }^{2}$ Department of Chemistry, University of Calcutta, \\ 92 A.P.C. Road, Kolkata 700 009, India
}

\begin{abstract}
We show that the delta function potential can be exploited along with perturbation theory to yield the result of certain infinite series. The idea is that any exactly soluble potential if coupled with a delta function potential remains exactly soluble. We use the strength of the delta function as an expansion parameter and express the second-order energy shift as an infinite sum in perturbation theory. The analytical solution is used to determine the second-order energy shift and hence the sum of an infinite series. By an appropriate choice of the unperturbed system, we can show the importance of the continuum in the energy shift of bound states.
\end{abstract}




\section{INTRODUCTION}

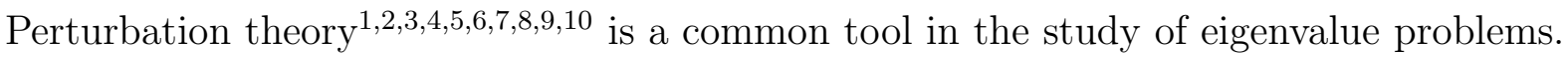
A classic eigenvalue problem is the time-independent Schrödinger equation, which can be written as

$$
H \psi=-\frac{\hbar^{2}}{2 m} \nabla^{2} \psi+V \psi=E \psi
$$

where $H$ is the Hamiltonian, $m$ is the mass of the particle, the wavefunction is $\psi$, and the eigenenergy is $E$. The problem is to find the allowed energies $E_{n}$ and the corresponding wavefunctions $\psi_{n}$. The energies $E_{n}$ are generally discrete and determined by the boundary conditions. Among the very few cases for which $E$ and $\psi$ can be determined exactly are a particle in a box, a particle confined by a harmonic potential, and a particle confined by a Coulomb potential. The shape-invariant potentials for which exact solutions can be found are variations on these cases. 11

We denote the Hamiltonian for which an exact solution can be found by $H_{0}$ and write an arbitrary Hamiltonian $H$ as

$$
H=H_{0}+\lambda H^{\prime}
$$

where $\lambda$ is a parameter and $H^{\prime}$ is the perturbing Hamiltonian. We denote the eigenvalues of $H_{0}$ by $E_{n}{ }^{(0)}$ and the eigenfunctions by $\psi_{n}^{(0)}$, and express the eigenvalues $E_{n}$ of $H$ in a power series expansion in $\lambda$ as $E_{n}=E_{n}^{(0)}+\lambda E_{n}^{(1)}+\lambda E_{n}^{(2)}+\ldots$ with

$$
\begin{aligned}
& E_{n}^{(1)}=\left\langle\psi_{n}^{(0)}\left|H^{\prime}\right| \psi_{n}^{(0)}\right\rangle, \\
& E_{n}^{(2)}=\sum_{l \neq n} \frac{\left|\left\langle\psi_{n}^{(0)}\left|H^{\prime}\right| \psi_{l}^{(0)}\right\rangle\right|^{2}}{E_{n}^{(0)}-E_{l}^{(0)}} .
\end{aligned}
$$

Equations (3) and (44) are valid when $\psi_{n}^{(0)}$ is nondegenerate. From Eq. (41) we see that the expression for $E_{n}^{(2)}$ is an infinite sum. If we choose the perturbation such that both $H$ and $H_{0}$ are exactly soluble, we can obtain an exact expression for $E_{n}^{(2)}$ which, when equated with the expression in Eq. (4), leads to an infinite series whose sum is known. 12

The $\delta$-function potential can be used to fulfill this purpose. The solutions of $H \psi=E \psi$ are the same as the solutions of $H_{0} \psi=E_{0} \psi$ except where the $\delta$-function is nonzero, and $E$ can be found by matching the solutions across the $\delta$-function. This procedure can provide some other insights as well. In Sec. II we discuss an example where the continuum states can change the sign of the contribution coming from the bound states. Another example is 
the effect of physical confinement on bound structures such as molecules. In this case we can study the effect of enclosing the $\delta$-function(s) between two infinite walls which is explored in Problem 1 in Sec. VI. A similar extension of Problem 3, given in Sec. VI is also possible.

In Sec. II we consider a box with infinite walls perturbed by an attractive $\delta$-function potential placed anywhere within the box. Next we consider a box of finite depth so that the number of bound states is finite. In this case the impact of the continuum states on the calculation of $E_{n}^{(2)}$ becomes of primary interest. In Sec. III we use the simple harmonic oscillator with a $\delta$-function potential at the origin to obtain an interesting infinite series and show how we can do the same for the one-dimensional hydrogen atom. The convergence of some other series is briefly considered in Sec. IV. We give a few suggested problems in Sec. VI.

\section{PARTICLE IN A BOX WITH DELTA FUNCTION(S)}

The $\delta$-function potential has been used as a model for atoms, molecules, solids, liquids, the many body problem, and scattering. $\underline{13}$ The usefulness of the $\delta$-function potential has been discussed by Lapidus ${ }^{14}$ in the context of stationary state perturbation theory. We place the potential at an arbitrary position, $x=p L$ with $0<p<1$, in a box of length $L$, with $0 \leq x \leq L$. The general solution of Eq. (1) can be written as

$$
\psi(x)= \begin{cases}A \sin k x+B \cos k x, & (0 \leq x \leq p L) \\ C \sin k x+D \cos k x, & (p L \leq x \leq L),\end{cases}
$$

where $k=\sqrt{2 m E / \hbar^{2}}$ and $A, B, C, D$ are determined from the normalization and boundary conditions. Because $\psi(x)=0$ at $x=0$, we have $B=0$. Hence we can write

$$
\psi(x)=A \sin k x, \quad(0 \leq x \leq p L)
$$

We also have $\psi(x=L)=0$ so that $D=-C \tan k L$ and

$$
\psi(x)=\tilde{C} \sin k(L-x), \quad(p L \leq x \leq L)
$$

where $\tilde{C}=C / \cos (k L)$. The continuity condition of the two functions at $x=p L$ gives

$$
\frac{A}{\tilde{C}}=\frac{\sin k(1-p) L}{\sin k p L}
$$


If we integrate Eq. (11) from $-\epsilon$ to $+\epsilon$ and take the limit as $\epsilon \rightarrow 0$

$$
\left.\frac{d \psi}{d x}\right|_{+\epsilon}-\left.\frac{d \psi}{d x}\right|_{-\epsilon}=\frac{2 m}{\hbar^{2}} \lim _{\epsilon \rightarrow 0} \int_{-\epsilon}^{+\epsilon} V(x) \psi(x) .
$$

It is clear that if $V(x)$ is finite, $\frac{d \psi}{d x}$ is continuous at $x=0$. But when $V(x)$ is infinite this agreement fails. In particular if $V(x)=-\lambda \delta(x)$

$$
\left.\frac{d \psi}{d x}\right|_{+\epsilon}-\left.\frac{d \psi}{d x}\right|_{-\epsilon}=-\frac{2 m \lambda}{\hbar^{2}} \psi(0) .
$$

Using this discontinuity condition at $x=p L$ and Eq. (8), we obtain the eigenvalue condition,

$$
k \sin k L=\frac{2 m \lambda}{\hbar^{2}} \sin k p L \sin k(1-p) L .
$$

If the perturbation is small, $k$ can be expanded as

$$
k=k^{(0)}+\lambda k^{(1)}+\lambda^{2} k^{(2)}+\ldots
$$

We write

$$
E_{n}=\frac{\hbar^{2}}{2 m} k^{2}=\frac{\hbar^{2}}{2 m}\left[k^{(0)}+\lambda k^{(1)}+\lambda^{2} k^{(2)}+\ldots\right]^{2}
$$

which gives

$$
\begin{aligned}
E_{n}^{(0)} & =\frac{\hbar^{2}}{2 m} k^{(0)^{2}}, \\
E_{n}^{(1)} & =\frac{\hbar^{2}}{2 m} 2 k^{(0)} k^{(1)}, \\
E_{n}^{(2)} & =\frac{\hbar^{2}}{2 m}\left[k^{(1)^{2}}+2 k^{(0)} k^{(2)}\right] .
\end{aligned}
$$

From Eqs. (11) and (12) we obtain

$$
\begin{aligned}
& k^{(0)}=\frac{n \pi}{L}, \\
& k^{(1)}=(-1)^{n} \frac{2 m}{k^{(0)} L \hbar^{2}} \sin ^{2}\left(k^{(0) p L}\right), \\
& k^{(2)}=-\frac{k^{(1)}}{k^{(0)}}+\frac{2 m k^{(1)}}{k^{(0)} \hbar^{2}}\left[\sin \left(k^{(0)} p L\right) \cos \left(k^{(0)} p L\right)(1-2 p)\right],
\end{aligned}
$$

so that $E_{n}^{(0)}=n^{2} \hbar^{2} \pi^{2} / 2 m L^{2}$ and

$$
\begin{aligned}
& E_{n}^{(1)}=-\frac{2}{L} \sin ^{2}(n \pi p), \\
& E_{n}^{(2)}=-\frac{2 m}{n^{2} \hbar^{2} \pi^{2}} \sin ^{4}(n \pi p)[1+2 \pi n(1-2 p) \cot (n \pi p)] .
\end{aligned}
$$


If we substitute the energy eigenvalues and eigenfunctions of the unperturbed Hamiltonian and write the perturbation as $H^{\prime}=-\lambda \delta(x-p L)$ in Eq. (41), we find

$$
E_{n}^{(2)}=\frac{8 m}{\hbar^{2} \pi^{2}} \sin ^{2}(n \pi p) \sum_{l \neq n} \frac{\sin ^{2}(l \pi p)}{n^{2}-l^{2}} .
$$

From Eqs. (16b) and (17), we find

$$
4 \sum_{l \neq n} \frac{\sin ^{2}(l \pi p)}{l^{2}-n^{2}}=\sin ^{2}(n \pi p)\left[\frac{1}{n^{2}}+2 \frac{\pi(1-2 p)}{n} \cot (n \pi p)\right] .
$$

If $l$ and $n$ are odd integers and $p=1 / 2$, Eq. (18) simplifies to

$$
4 \sum_{l \neq n} \frac{1}{l^{2}-n^{2}}=\frac{1}{n^{2}} \quad(l, n \text { odd }) .
$$

Equation (19) is identical to Eq. $\left(A_{3}\right)$ of Ref. 14. To verify that the sum is correct and the series is convergent, we write Eq. (19) as

$$
\begin{aligned}
4 \sum_{l \neq n} \frac{1}{l^{2}-n^{2}} & =\frac{2}{n} \lim _{N \rightarrow \infty}\left[\sum_{m \neq \frac{n-1}{2}}^{N} \frac{1}{(2 m+1)-n}-\sum_{m \neq \frac{n-1}{2}}^{N} \frac{1}{(2 m+1)+n}\right] \\
& =\frac{1}{n^{2}}+\lim _{N \rightarrow \infty}\left[\sum_{\frac{1-n}{2}}^{-1} \frac{1}{p}+\sum_{-1}^{\frac{n-1}{2}} \frac{1}{p}+\sum_{\frac{n+1}{2}}^{N-\frac{n+1}{2}} \frac{1}{p}-\sum_{\frac{n+1}{2}}^{N+\frac{n+1}{2}} \frac{1}{q}\right] \\
& =\frac{1}{n^{2}}+\lim _{N \rightarrow \infty}\left[\sum_{\frac{n+1}{2}}^{N-\frac{n+1}{2}} \frac{1}{p}-\sum_{\frac{n+1}{2}}^{N+\frac{n+1}{2}} \frac{1}{q}\right]=\frac{1}{n^{2}}
\end{aligned}
$$

because for finite $n, N-(n-1) / 2$ and $N+(n+1) / 2$ both approach $N$ as $N \rightarrow \infty$.

We can employ the first-order correction term to the wavefunction, which also has a sum-over-states form, to obtain a sum rule. We have

$$
\psi(x)= \begin{cases}A \sin k x, & (0 \leq x \leq p L) \\ A \frac{\sin k p L}{\sin k(1-p) L} \sin k(L-x) & (p L \leq x \leq L)\end{cases}
$$

with

$$
A^{2}\left[\frac{L}{2}-\frac{\sin k L \cos 2 k L(1-2 p)}{2 k}\right]=1
$$

from the normalization condition. If we write $k$ as in Eq. (12) and write $\psi=\psi^{(0)}+\lambda \psi(1)+\ldots$, the first-order shift in the wavefunction can be found from

$$
\begin{aligned}
\psi(x) & =\frac{\sin k^{(0)} x+\lambda k^{(1)} x \cos k^{(0)} x}{\sqrt{\frac{L}{2}}\left[1-\frac{\lambda k^{(1)} L}{k^{(0)} L} \cos k^{(0)} L \cos 2 k^{(0)} L(1-2 p)\right]^{1 / 2}} \\
& \approx \sqrt{\frac{2}{L}} \sin k^{(0)} x\left[1+\frac{\lambda k^{(1)}}{2 k^{(0)}}(-1)^{n} \cos 4 n p \pi\right]+\lambda \sqrt{\frac{2}{L}} k^{(1)} x \cos k^{(0)} x+O\left(\lambda^{2}\right)
\end{aligned}
$$


Thus the first-order shift $\psi_{n}^{(1)}(x)$ is

$$
\psi_{n}^{(1)}(x)=\sqrt{\frac{2}{L}} \sin \frac{n \pi x}{L} \frac{k^{(1)}}{2 k^{(0)}}(-1)^{n} \cos 4 n p \pi+\sqrt{\frac{2}{L}} k^{(1)} x \cos \frac{n \pi x}{L} .
$$

If we use the fact that $H_{l n}^{\prime}=-\frac{2}{L} \sin n p \pi \sin l p \pi$, where $H_{l n}^{\prime}=\left\langle\psi_{l}^{(0)}|H| \psi_{n}^{(0)}\right\rangle$, we can write Eq. (3) as

$$
\psi_{n}^{(1)}(x)=-\sqrt{\frac{2}{L}} \frac{2}{L} \sum_{l \neq n} \frac{\sin n p \pi \sin l p \pi \sin \frac{l \pi x}{L}}{E_{n}^{(0)}-E_{l}^{(0)}},
$$

which leads to

$$
\begin{aligned}
& -\sqrt{\frac{2}{L}} \frac{2}{L} \sum_{l \neq n} \frac{\sin n p \pi \sin l p \pi \sin \frac{l \pi x}{L}}{\frac{\pi^{2} \hbar^{2}}{2 m L^{2}}\left(n^{2}-l^{2}\right)}=\sqrt{\frac{2}{L}} \sin \frac{n \pi x}{L} \frac{k^{(1)}}{2 k^{(0)}}(-1)^{n} \cos 4 n p \pi \\
& +\sqrt{\frac{2}{L}} k^{(1)} x \cos \frac{n \pi x}{L},
\end{aligned}
$$

with $l$ and $n$ odd and $0 \leq x \leq p L$.

The effect of continuum states in second-order perturbation theory has been considered in Refs. 15, 16,17,18,19. A one-dimensional attractive $\delta$-function potential was considered in Ref. 17. This potential supports only one bound state $\underline{20}$ and for a given perturbation, the contribution to $E_{n}^{(2)}$ comes only from the continuum states. A one-dimensional square well potential perturbed by another square well potential was discussed in Ref. 18.

In this context we discuss an example where the result of $E_{n}^{(2)}$ is a sum of a finite series for a finite number of bound states and an integral from the continuum. The continuum contribution is so large that it reverses the sign of the sum. We consider a finite square well potential, $V=0$ for $-L<x<L$ and $V=V_{0}$ outside for which there is a finite number of bound states determined by the magnitude of $V_{0} \cdot \frac{5,20,21}{}$ In the following we will consider the bound state with the highest energy. The perturbation is an attractive $\delta$-function at $x=0$. Because all the other bound states are lower in energy than the one we are considering, their contribution to the sum in Eq. (4) is positive.

Equation (11) outside the well can be written as

$$
\frac{d^{2}}{d x^{2}} \psi(x)+\kappa^{2} \psi(x)=0,
$$

with

$$
\kappa^{2}=\frac{2 m}{\hbar^{2}}\left(V_{0}-E\right)
$$


The corresponding solution is

$$
\psi(x)= \begin{cases}B \exp (-\kappa x) & (x>0) \\ B \exp (\kappa x) & (x<0)\end{cases}
$$

Equation (11) inside the well can be written as

$$
\frac{d^{2}}{d x^{2}} \psi(x)+k^{2} \psi(x)=0,
$$

where

$$
k^{2}=\frac{2 m E}{\hbar^{2}}
$$

The corresponding solutions are

$$
\psi(x)= \begin{cases}A \cos k x, & \text { even } \\ A \sin k x, & \text { odd }\end{cases}
$$

The acceptability conditions for the wavefunctions are equivalent to requiring that the logarithmic derivative of the wavefunction be continuous at $x= \pm L$. For even states this condition leads to

$$
\begin{aligned}
\tan k L & =\frac{\kappa}{k}=\sqrt{\frac{\alpha^{2} L^{2}}{k^{2} L^{2}}-1} \\
\alpha & =\sqrt{\frac{2 m V_{0}}{\hbar^{2}}} .
\end{aligned}
$$

If we plot $\alpha^{2} L^{2}-k^{2} L^{2}$ and $\sqrt{k^{2} L^{2}} \tan ^{2} \sqrt{k^{2} L^{2}}$ versus $k^{2} L^{2}$, the intersections will give the scaled values of the bound state energies. Here all bound states are non-degenerate. Even and odd solutions alternate as the energy increases. The number of bound states is finite and equal to $N+1$ if $N \pi<2 \alpha L \leq(N+1) \pi$. Hence, by selecting a value of $\alpha$, that is, $V_{0}$, we can control the number of bound states.

If we perturb the system by placing an attractive $\delta$-function potential at $x=0$, the wavefunction is given as

$$
\psi(x)= \begin{cases}A \cos (k x)+B \sin k x & (0<x \leq L) \\ A \cos (k x)-B \sin k x & (0>x \geq-L) \\ C \exp (-\kappa|x|) & (x>|L|)\end{cases}
$$


The continuity condition at $x=0$ gives $B=m \lambda A / k \hbar^{2}$, and the wavefunction inside the well is

$$
\psi(x)=A\left[\cos k x+\frac{m \lambda}{k \hbar^{2}} \sin k x\right] .
$$

The continuity condition at $x=+L$ gives

$$
k^{2} \tan k L-k \kappa=\lambda \frac{m}{\hbar^{2}}[\kappa \tan k L+k] .
$$

If we substitute the values of $k$ and $\kappa$ given in Eqs. (32) and (29) in Eq. (37), we obtain the desired result

$$
E \tan \left(\sqrt{2 m E} \frac{L}{\hbar}\right)-\sqrt{E\left(V_{0}-E\right)}=\lambda \frac{\sqrt{m}}{\sqrt{2} \hbar}\left[\sqrt{V_{0}-E} \tan \left(\sqrt{2 m E} \frac{L}{\hbar}\right)+\sqrt{E}\right] .
$$

We expand $E$ in powers of $\lambda$, equate terms of same order of $\lambda$ on both sides and obtain

$$
\begin{aligned}
\tan \left(\sqrt{2 m E_{n}^{(0)}} \frac{L}{\hbar}\right) & =\sqrt{\frac{\epsilon}{E_{n}^{(0)}}}, \\
E_{n}^{(1)} & =\frac{\sqrt{\frac{2 m \epsilon}{\hbar}}}{1+L \sqrt{\frac{2 m \epsilon}{\hbar}}}, \\
E_{n}^{(2)} & =-\frac{V_{0}}{E_{n}^{(0)^{3 / 2}}} \frac{1}{\frac{L V_{0} \sqrt{m}}{\hbar \sqrt{2 E_{n}^{(0)}}}+\frac{1}{2} \sqrt{\frac{\epsilon}{E_{n}^{(0)}}}+\frac{1}{2} \sqrt{\frac{E_{n}^{(0)}}{\epsilon}}} \\
& \times \frac{\frac{1}{2} m^{3 / 2} L \epsilon+\frac{m^{2} L^{2} \epsilon^{3 / 2}}{\sqrt{2} \hbar}+\frac{m \hbar \epsilon}{2 \sqrt{2}}\left(2 E_{n}^{(0)}-V_{0}\right)+\frac{m^{3 / 2} L \epsilon}{2}\left(2 E_{n}^{(0)}-V_{0}\right)}{\sqrt{2} \hbar^{2}\left[\hbar+L \sqrt{2 m \epsilon}\left[1+L^{2} \frac{2 m \epsilon}{\hbar^{2}}+\frac{2 L}{\hbar} \sqrt{2 m \epsilon}\right]\right.}
\end{aligned}
$$

where $\epsilon=V_{0}-E_{n}^{(0)}$. For the $n$th state to be bound $E_{n}^{(0)}$ must be less than $V_{0}$. From the solution for $E_{n}^{(0)}$ from Eq. (39a) we see that $E_{n}^{(0)} \leq \frac{1}{2} V_{0}$, even for the most excited bound state. Given these constraints, we conclude that $E_{n}^{(2)}<0$, which makes it clear that the integral from the continuum states has a greater contribution than the discrete sum. If we fix $V_{0}$ and $L$ such that there are only three bound states with energies $E_{1}, E_{2}$, and $E_{3}$, then wavefunctions corresponding to $E_{1}$ and $E_{3}$ are even and that corresponding to $E_{2}$ is odd. The contribution from the bound states to the energy shift of $E_{3}$ to second order is

$$
E_{3}^{(2)}=\frac{A_{1} A_{3}}{E_{3}-E_{1}}
$$

where $A_{1}$ and $A_{3}$ are defined in Eq. (35) for the ground state and the second excited state respectively with a similar meaning for $k_{1}$ and $k_{3}$. The relation between $A$ and $k$ is given by

$$
A_{1,3}=\frac{\sqrt{2}}{\sqrt{L \pm \frac{1}{2 k} \sin \left(2 k_{1,3} L\right)+\cos ^{2}\left(\frac{k_{1,3} L}{2 \kappa}\right)}},
$$


which is positive, where + and - signs are for $A_{1}$ and $A_{3}$ respectively. The contribution from the continuum makes the final answer as given by Eq. (39c) and is negative, showing the important role of the continuum.

\section{PARTICLE CONFINED BY A HARMONIC POTENTIAL}

We now consider the exactly soluble system of a simple harmonic oscillator perturbed by an attractive delta potential at the origin. The unperturbed Hamiltonian is $H_{0}=p^{2} / 2 m+$ $k x^{2} / 2$, and the perturbation is $H^{\prime}=-\lambda \delta(x)$. Equation (1) for the unperturbed system can be solved as

$$
\frac{d^{2}}{d z^{2}} \psi(z)+\left(n+\frac{1}{2}-\frac{z^{2}}{4}\right) \psi(z)=0
$$

where $z=\sqrt{2 m \omega / \hbar} x$ and $E_{n}=(n+1 / 2) \hbar \omega$, subject to the boundary condition that the wavefunction vanishes at $\pm \infty$. The even and odd solutions of the unperturbed system are

$$
\begin{aligned}
& y_{1}=e^{-z^{2} / 4}{ }_{1} F_{1}\left(\frac{a}{2}+\frac{1}{4} ; \frac{1}{2} ; \frac{z^{2}}{2}\right) \text { even, } \\
& y_{2}=z e^{-z^{2} / 4}{ }_{1} F_{1}\left(\frac{a}{2}+\frac{3}{4} ; \frac{3}{2} ; \frac{z^{2}}{2}\right) \text { odd },
\end{aligned}
$$

where the true solution $\psi$ is a linear combination of $y_{1}$ and $y_{2}$ (see Eq. (47)) and ${ }_{1} F_{1}$ is the confluent hypergeometric function (the Kummer function). Its power series expansion can be written as

$$
{ }_{1} F_{1}(a ; b ; z)=1+\frac{a}{b} \frac{z}{1 !}+\frac{a(a+1)}{b(b+1)} \frac{z^{2}}{2 !}+\ldots
$$

The solution can be written in the usual form as

$$
\begin{aligned}
& U(a, z)=\cos \pi\left(\frac{1}{4}+\frac{a}{2}\right) Y_{1}-\sin \pi\left(\frac{1}{4}+\frac{a}{2}\right) Y_{2}, \\
& V(a, z)=\frac{1}{\Gamma\left(\frac{1}{2}-a\right)}\left[\sin \pi\left(\frac{1}{4}+\frac{a}{2}\right) Y_{1}-\cos \pi\left(\frac{1}{4}+\frac{a}{2}\right) Y_{2}\right],
\end{aligned}
$$

where $Y_{1}$ and $Y_{2}$ are given by

$$
\begin{aligned}
& Y_{1}=\frac{1}{\sqrt{\pi}} \frac{\Gamma\left(\frac{1}{4}-\frac{a}{2}\right)}{2^{\left(\frac{a}{2}+\frac{1}{4}\right)}} y_{1}, \\
& Y_{2}=\frac{1}{\sqrt{\pi}} \frac{\Gamma\left(\frac{3}{4}-\frac{a}{2}\right)}{2^{\left(\frac{a}{2}+\frac{1}{4}\right)}} y_{2} .
\end{aligned}
$$

We write the general solution for the perturbed problem as

$$
\psi= \begin{cases}A y_{1}+B y_{2} & (x>0) \\ C y_{1}+D y_{2} & (x<0)\end{cases}
$$


These two forms of $\psi$ must match at $x=0$, giving $A=C$. The wavefunction must be either symmetric or antisymmetric. The antisymmetric functions remain unchanged even after introducing the delta potential. The symmetric functions must change and are expressed as

$$
\psi=\left\{\begin{array}{l}
A y_{1}+B y_{2}, \\
A y_{1}-B y_{2} .
\end{array}\right.
$$

The discontinuity condition of the $\delta$-function at the origin gives $B=-m \lambda \xi A / \hbar^{2}$, where $\xi=\sqrt{\hbar / 2 m \omega}$, and the acceptable wavefunction takes the form

$$
\psi=A\left[y_{1}-\frac{m \lambda \xi}{\hbar^{2}} y_{2}\right]
$$

The boundary condition $\psi \rightarrow 0$ as $x \rightarrow \infty$ leads to

$$
y_{1}-\frac{m \lambda \xi}{\hbar^{2}} y_{2}=0
$$

If we compare this form with Eq. (45a), we find that $U(a, x) \rightarrow 0$ as $x \rightarrow \infty$ and thus we obtain

$$
\sqrt{2} \frac{\Gamma\left(\frac{3}{4}-\frac{a}{2}\right)}{\Gamma\left(\frac{1}{4}-\frac{a}{2}\right)} \tan \left(\frac{\pi}{4}+\frac{\pi a}{2}\right)=-\frac{m \lambda \xi}{\hbar^{2}} .
$$

We express $a$ and $n$ in terms of $E_{n}$ and expand the latter in powers of $\lambda$ and find

$$
\begin{aligned}
& E_{n}^{(0)}=\left(2 n+\frac{1}{2}\right) \hbar \omega \\
& E_{n}^{(1)}=-\frac{1}{\sqrt{\pi}} \sqrt{\frac{m \omega}{\hbar} \frac{\Gamma\left(n+\frac{1}{2}\right)}{\Gamma(n+1)}} \\
& E_{n}^{(2)}=-\frac{m}{2 \pi^{2} \hbar^{2}}\left[\frac{\Gamma\left(n+\frac{1}{2}\right)}{\Gamma(n+1)}\right]^{2}\left[\psi(n+1)-\psi\left(n+\frac{1}{2}\right)\right],
\end{aligned}
$$

where

$$
\begin{aligned}
\psi(n+1) & =\frac{\Gamma^{\prime}(n+1)}{\Gamma(n+1)}, \\
\psi\left(n+\frac{1}{2}\right) & =\frac{\Gamma^{\prime}\left(n+\frac{1}{2}\right)}{\Gamma\left(n+\frac{1}{2}\right)} .
\end{aligned}
$$

On the other hand, if we substitute the values of the energy eigenvalues and eigenfunctions of the unperturbed Hamiltonian in Eq. (4), we can express $E_{n}^{(2)}$ as

$$
E_{n}^{(2)}=\frac{1}{2 \pi} \frac{m}{\hbar^{2}} \sum_{l \neq n}\left[\frac{1}{2^{2(n+l)}} \frac{(2 l) !(2 n) !}{(l !)^{2}(n !)^{2}} \frac{1}{(n-l)}\right] .
$$


From Eqs. (52c) and (154), we find

$$
\sum_{l \neq n}\left[\frac{1}{2^{2(n+l)}} \frac{(2 l) !(2 n) !}{(l !)^{2}(n !)^{2}} \frac{1}{(n-l)}\right]=-\frac{1}{\pi^{2}}\left[\frac{\Gamma\left(n+\frac{1}{2}\right)}{\Gamma(n+1)}\right]^{2}\left[\psi(n+1)-\psi\left(n+\frac{1}{2}\right)\right],
$$

which is a new series with a known sum at the right hand side and may be of mathematical interest.

We end this section with a brief look at the one-dimensional hydrogen atom. In this case we place the $\delta$-function potential at $x=a$, so that the Hamiltonian is

$$
H=-\frac{\hbar^{2}}{2 m} \frac{d^{2}}{d x^{2}}-\frac{e^{2}}{|x|}-\lambda \delta(x-a)
$$

The eigenvalue equation is with $E=-|E|$

$$
\frac{d^{2} \psi}{d \rho^{2}}+\frac{2 \alpha}{\rho} \psi+\lambda \sqrt{\frac{2 m}{\hbar^{2}|E|}} \delta(\rho-k a) \psi=\psi
$$

where $\alpha=e^{2} \sqrt{m / 2 \hbar^{2}\left|E_{0}\right|}=e^{2} m / \hbar^{2} k$. The wavefunction is continuous at $\rho=k a$ and

$$
\left.\frac{d \psi}{d \rho}\right|_{+}-\left.\frac{d \psi}{d \rho}\right|_{-}=-\lambda \sqrt{\frac{2 m}{\hbar^{2}|E|}} \psi
$$

at $\rho=k a$. For $\rho<k a, \psi$ is well behaved at the origin and vanishes at infinity. We have

$$
\psi= \begin{cases}A \rho e^{-\rho} M(1-\alpha, 2,2 \rho), & \rho<k a \\ \psi=B \rho e^{-\rho} U(1-\alpha, 2,2 \rho) & \rho>k a .\end{cases}
$$

Here

$$
M(a, b, z)=1+\frac{a z}{b}+\frac{(a)_{2} z^{2}}{(b)_{2}}+\ldots
$$

and

$$
\begin{aligned}
U(1-\alpha, 2,2 \rho) & =\lim _{\epsilon \rightarrow 0} U(1-\alpha, 2+\epsilon, 2 \rho) \\
& =\lim _{\epsilon \rightarrow 0} \frac{\pi}{\sin \pi \epsilon}\left[\frac{M(1-\alpha, 2,2 \rho)}{\Gamma(-\alpha)}-\frac{1}{2 \rho} \frac{M(-\alpha,-\epsilon, 2 \rho)}{\Gamma(1-\alpha) \Gamma(-\epsilon)}\right]
\end{aligned}
$$

and $(\beta)_{n}=\beta(\beta+1)(\beta+2) \cdots(\beta+n+1)$. We find the eigenvalue equation

$$
\begin{aligned}
-M(1-\alpha, 2,2 k a) U^{\prime}(1-\alpha, 2,2 k a) & +M^{\prime}(1-\alpha, 2,2 k a) U(1-\alpha, 2,2 k a) \\
& =\frac{m \lambda}{\hbar^{2} k} M(1-\alpha, 2,2 k a) U(1-\alpha, 2,2 k a) .
\end{aligned}
$$


Now we can proceed to find the second-order energy shift. Although it is not instructive to show more details, outlining the procedure for the hydrogen atom is useful because it is a prototype for problems in higher dimension. In such situations, if we want to perturb the exactly soluble model by a $\delta$-function, it is necessary to put the one dimensional $\delta$-function at some $r>0$ rather than at $r=0$, where it has no influence.

\section{DISCUSSION}

To obtain a feeling for the convergence properties of the series that we have found, we present some numerical results. We first consider Eq. (19) and rewrite it in the form

$$
\sum_{l \neq n} \frac{1}{l^{2}-n^{2}}=\frac{1}{4 n^{2}} \quad(l, n \text { odd })
$$

Table I shows how the sum on the left approaches $1 / 4 n^{2}$ in two sample cases, e.g. at $n=1$ and $n=5$, with increasing number of terms. We can generate other series of this sort by taking other values of $p\left(\neq \frac{1}{2}\right)$ in Eq. (18). It is also interesting to determine what happens to the sum in Eq. (63) if the restrictions on $l$ and $n$ are removed. We can easily find that such a series converges to $-1 / 4 n^{2}$ :

$$
\sum_{l=0(\neq n)} \frac{1}{l^{2}-n^{2}}=-\frac{1}{4 n^{2}} \quad(n \neq 0) .
$$

To verify, one may proceed as follows:

$$
\begin{aligned}
\sum_{l=0(\neq n)} \frac{1}{l^{2}-n^{2}} & =\lim _{N \rightarrow \infty}\left(\sum_{l=0}^{n-1} \frac{1}{l^{2}-n^{2}}+\sum_{l=n+1}^{N} \frac{1}{l^{2}-n^{2}}\right) \\
& =\frac{1}{2 n} \sum_{l=0}^{n-1}\left(\frac{1}{l-n}-\frac{1}{l+n}\right)+\frac{1}{2 n} \lim _{N \rightarrow \infty} \sum_{l=n+1}^{N}\left(\frac{1}{l-n}-\frac{1}{l+n}\right) \\
& =-\frac{1}{2 n} \sum_{j=1}^{n} \frac{1}{j}-\frac{1}{2 n} \sum_{j=n}^{2 n-1} \frac{1}{j}+\frac{1}{2 n} \lim _{N \rightarrow \infty}\left(\sum_{j=1}^{N-n} \frac{1}{j}-\sum_{j=2 n+1}^{N+n} \frac{1}{j}\right) \\
& =-\frac{1}{2 n} \frac{1}{n}-\frac{1}{2 n} \sum_{j=1}^{2 n-1} \frac{1}{j}+\frac{1}{2 n} \lim _{N \rightarrow \infty}\left(\sum_{j=1}^{N-n} \frac{1}{j}-\sum_{j=2 n+1}^{N+n} \frac{1}{j}\right) \\
& =-\frac{1}{2 n^{2}}+\frac{1}{4 n^{2}}+\frac{1}{2 n} \lim _{N \rightarrow \infty}\left(\sum_{j=1}^{N-n} \frac{1}{j}-\sum_{j=1}^{N+n} \frac{1}{j}\right) \\
& =-\frac{1}{4 n^{2}},
\end{aligned}
$$


as, at the limit $N \rightarrow \infty, N \pm n \approx N$. Consequently, the terms within parentheses tend to zero.

We now turn our attention to Eq. (27) which simplifies to the form

$$
\sum_{l \neq n} \frac{\sin l p \pi \sin \frac{l \pi x}{L}}{\left(l^{2}-n^{2}\right)}=\frac{1}{4 n^{2}} \sin n p \pi \cos 4 n p \pi \sin \frac{n \pi x}{L}-\frac{\pi x}{2 n L} \sin n p \pi \cos \frac{n \pi x}{L},
$$

where $l$ and $n$ are odd integers, $0 \leq p \leq 1$ and $0 \leq x \leq p L$. Unlike Eq. (18), there is an additional variable $x$ so that we can find many interesting infinite series with closed form answers. For example, we consider $p=1 / 2, x=L / 4$, and $n=1$ in Eq. (65) that yields

$$
\frac{1}{3^{2}-1}+\frac{1}{5^{2}-1}-\frac{1}{7^{2}-1}-\frac{1}{9^{2}-1}+\frac{1}{11^{2}-1}+\frac{1}{13^{2}-1}+\ldots=\frac{\pi-2}{8} .
$$

If we group terms that are adjacent and have the same sign, we obtain

$$
\sum_{k=1}^{\infty} \frac{-1^{k+1}}{4 k}\left(\frac{1}{4 k-2}+\frac{1}{4 k+2}\right)=\frac{\pi-2}{8}
$$

with no restriction on $k$. This series is interesting because it shows a sawtooth convergence. Alternate partial sums provide upper and lower bounds, which become gradually closer. For such series, it is well known that averaging two adjacent terms leads to better convergence. Table II shows some results for $\pi$ with a small number of terms in Eq. (67). We denote the sums by $S(j)$ and construct $\overline{S(j)}=\frac{[S(j)+S(j-1)]}{2}$ to see how the average sum approaches better the desired value. By increasing the number of terms in Eq. (67), we can obtain very good estimates of $\pi$ as shown in Table III. Note that the series (66) or (67) is not commonly used to estimate $\pi$. The well known Gregory-Leibniz series converges very slowly 24 . Many other

series for $\pi$ are available with varying convergence properties (see, for example, Ref. 24). Series (67) may be a useful addition to such a list.

We note finally that the infinite sums in Eqs. (18) and (67) for specific values of the parameters such as $p$ and $n$ or the variable $x$, have a common property: They have the same asymptotic behavior as the series (19). Hence, we can be sure about their convergence, in view of Eq. (201).

\section{CONCLUSION}

Exactly soluble potentials remain exactly soluble when we add a $\delta$-function or a set of $\delta$-functions (see Problem 3 in Sec. VI for an example). In the three cases we studied, the 
problem can be solved in two different ways. An exact solution can be obtained by joining the piecewise exact results for the wavefunction at the $\delta$-discontinuities. Or we can use perturbation theory in the strength of the $\delta$-function to solve the problem to any desired order. Because the second-order perturbation theory result is usually in the form of an infinite series, we can use the exact solutions to determine the sums of such series, and exhibit their convergence properties. We can consider other exactly soluble potentials as well.22 New infinite sums with known closed form answers may be found. Recently, an alternative scheme has been put forward using Green's function techniques. ${ }^{23}$

By choosing appropriate examples, we can sometimes get insight into physics of certain problems that are not usually considered. The role of the continuum in the second order shift for the finite well is a case in point. In Problem 1 we explore the effect of a single attractive $\delta$-function. We have seen that below a critical length, the bound state of the $\delta$-function disappears. Two attractive $\delta$-functions can be used to set up a toy model for the hydrogen molecular ion. Placing walls in this case on the two sides allows us to study the effect of confinement on the binding of a molecule. We can imagine carrying this further and placing a periodic array of $\delta$-functions interrupted on each side by a wall. The resulting violation of Bloch's theorem would make an interesting study.

\section{SUGGESTED PROBLEMS}

Problem 1. In Sec. II we considered only the positive eigenenergies for the $\delta$-function potential in a box. The attractive $\delta$-function by itself will have a negative energy bound state. In this problem we explore what happens when the $\delta$-function is placed in a box. Consider a $\delta$-function at the center of a box of width $2 L$. Repeat the calculation of Sec. II, but for a negative energy bound state and show that for a $\delta$-function of strength $\lambda$, the negative energy bound state will disappear if $L$ is smaller than $L_{c}=\hbar^{2} / m \lambda$.

Problem 2. Consider the perturbation of a particle confined in a one-dimensional box of length $L$ by an attractive $\delta$-function potentials at $x=p L$ with $0<p<1$. The wavefunction is given as

$$
\psi(x)= \begin{cases}A \sin k x & (0 \leq x \leq p L) \\ A \frac{\sin k p L}{\sin k(1-p) L} \sin k(L-x) & (p L \leq x \leq L),\end{cases}
$$

where $A$ is a normalization constant. Explore the wavefunction in the region $p L \leq x \leq L$ 
to obtain the first order correction to wavefunction. Also derive the same from perturbation theory and show that this leads to an interesting sum rule. Is the series convergent?

Problem 3. Consider a particle confined to a one-dimensional box of length $L$ perturbed by two attractive $\delta$-function potentials at $x=p L$ and $x=q L$ with $0<p<1$ and $0<q<1$. (a) Find the second order energy shift and show that it is always negative when $p=\frac{1}{4}$ and $q=\frac{3}{4}$. (b) Do the same using Rayleigh-Schrödinger perturbation theory and compare the result with the above. Does this series converge? (c) Find the first order correction to the wavefunction and show how this correction gives three important sum rules which are convergent.

Problem 4. Consider a simple harmonic oscillator and perturb it by an attractive delta potential at the origin. Use perturbation theory to find the first order correction to the wavefunction and show that this correction leads to another sum rule.

\section{Acknowledgments}

We are thankful to an anonymous referee for many constructive suggestions. NB would like to thank the Council of Scientific and Industrial Research, India for a research fellowship.

* Electronic address: tpjkb@iacs.res.in

1 A. Dalgarno, "Stationary perturbation theory," in Quantum theory, edited by D. R Bates (Academic Press, New York, 1961), Vol. I, pp. 171-209.

2 J. O. Hirschfelder, W. Byers-Brown, and S. T. Epstein, "Recent developments in perturbation theory," Adv. Quantum Chem. 1, 255-374 (1964).

3 C. H. Wilcox, Perturbation Theory and its Applications in Quantum Mechanics (John Wiley \& Sons, NY, 1966).

4 M. Reed and B. Simon, Methods of Modern Mathematical Physics (Academic Press, New York, 1978), Vol. 4.

5 D. J. Griffiths, Introduction to Quantum Mechanics (Pearson, Singapore, 2006), 2nd ed.

6 B. H. Bradsten and C. J. Joachain, Quantum Mechanics (Pearson, Singapore, 2004), 2nd ed.

7 C. K. Au, C. K. Chow, and C. S. Chu, "A new efficient method for calculating perturbative 
energies using functions which are not square integrable: Regularization and justification," J. Phys. A 30, 4133-4136 (1997).

8 W. Scherer "Quantum averaging II. Kolmogorov's algorithm," J. Phys. A 30, 2825-2851 (1997).

9 W. Hai, M. Feng, X. Zhu, L. Shi, K. Gao, X. Fang, and G. Chong, "Numerical instability in Rayleigh-Schrodinger quantum mechanics," J. Phys. A 34, L79-L87 (2001).

10 J. P. Killingbeck and G. Jolicard, "The Bloch wave operator: Generalization and application: Part I. The time-independent case," J. Phys. A 36, R105-R180 (2003).

11 F. Cooper, A. Khare, and U. Sukhatme, Supersymmetry in Quantum Mechanics (World Scientific, Singapore, 2001).

12 B. Simon, "Coupling constant analyticity for the anharmonic oscillator," Ann. Phys. (NY) 58, 76-136 (1970).

13 A large number of references in these fields can be found in I. R. Lapidus, "Relativistic onedimensional hydrogen atom", Am. J. Phys. 51, 1036-1038 (1983); "Bound states and scattering from a one-dimensional hydrogen atom at a boundary" ibid., 51, 1137-1139 (1983).

14 I. R. Lapidus, "Particle in a square well with a $\delta$-function perturbation," Am. J. Phys. 55, $172-174$ (1987).

15 L. I. Schiff, Quantum Mechanics (McGraw-Hill, New York, 1968), 3rd ed.

16 L. D. Landau and E. M. Lifshitz, Quantum Mechanics, Non-relativistic Theory (ButterworthHeinemann 2005), 3rd ed.

17 D. Kiang, "Contribution of the continuum in perturbation theory," Am. J. Phys. 45, 308-309 (1977) and references therein.

18 M. Coronado, N. Dominguez, J. Flores, and C. de la Portilla, "Effect of the continuum states in second-order perturbation theory: An example," Am. J. Phys. 50, 27-29(1982).

19 V. Urumov and G. J. Ivanovski, "The role of continuum states in perturbation theory," Am. J. Phys. 51, 950-952 (1983).

20 E. Merzbacher, Quantum Mechanics (John Wiley \& Sons, Singapore 2004), 3rd ed.

21 O. F. de Alcantara Bonfim and D. J. Griffiths, "Exact and approximate energy spectrum for the finite square well and related potentials," Am. J. Phys 74, 43-48 (2005).

22 J. N. Pronchik and B. W. Williams, "Exactly solvable quantum mechanical potentials: An alternative approach," J. Chem. Educ. 80, 918-926 (2003).

23 C. V. Sukumar, "Sum rules for confining potentials," arXiv: quant-ph/0611066, November 
(2006).

24 See for example, $\langle$ mathworld.wolfram.com $\rangle$, 


\section{Tables}

\begin{tabular}{|c|c|c|}
\hline No. of terms & Results for $n=1$ & Results for $n=5$ \\
\hline 10 & 0.2273 & -0.013 \\
\hline 50 & 0.2451 & 0.0051 \\
\hline 100 & 0.2475 & 0.0075 \\
\hline 1000 & 0.2498 & 0.00975 \\
\hline 10000 & 0.249975 & 0.009975 \\
\hline 100000 & 0.2499975 & 0.0099975 \\
\hline
\end{tabular}

TABLE I: Convergence study of Eq. (63) in two sample cases

\begin{tabular}{|c|c|c|}
\hline $\begin{array}{c}\text { No. of terms } \\
{[j]}\end{array}$ & $\begin{array}{c}\text { Results for } \pi \\
{[S(j)]}\end{array}$ & $\begin{array}{c}\text { Average estimate } \\
\overline{[S(j)]}\end{array}$ \\
\hline 1 & 3.33 & \\
\hline 2 & 3.07 & 3.20 \\
\hline 3 & 3.18 & \\
\hline 4 & 3.12 & 3.149 \\
\hline 5 & 3.16 & \\
\hline 6 & 3.13 & 3.144 \\
\hline 7 & 3.15 & \\
\hline 8 & 3.13 & 3.143 \\
\hline 9 & 3.147 & \\
\hline 10 & 3.137 & 3.142 \\
\hline 19 & 3.1429 & \\
\hline 20 & 3.1404 & 3.1417 \\
\hline
\end{tabular}

TABLE II: Convergence study of Eq. (67) for estimates of $\pi$ 


\begin{tabular}{|c|c|}
\hline$j$ & $\overline{S(j)}$ \\
\hline 10 & 3.142 \\
\hline 100 & 3.141593 \\
\hline 1000 & 3.141592654 \\
\hline 10000 & 3.14159265359 \\
\hline 100000 & 3.1415926535897938 \\
\hline
\end{tabular}

TABLE III: Gradually improved estimates of $\pi$ from Eq. (67) 